\begin{tabular}{|c|c|c|}
\hline $\begin{array}{l}\text { PKS } \\
\text { PUBLIC } \\
\text { KNDOLEDGE } \\
\text { PROJECT }\end{array}$ & $\begin{array}{l}\text { Revista de GEOGRAFIA } \\
\text { (RECIFE) } \\
\text { http://www.revista.ufpe.br/revistageografia }\end{array}$ & $\begin{array}{l}\text { OJS } \\
\text { OPEN } \\
\text { JORNAL } \\
\text { SYSTEMS }\end{array}$ \\
\hline
\end{tabular}

\title{
GEOMORFOLOGIA FLUVIAL NO SEMIÁRIDO BRASILEIRO
}

\author{
Andrea Almeida Cavalcante ${ }^{1}$ \\ ${ }^{1}$ Universidade Estadual do Ceará.E-mail: andrea.cavalcante@uece.br
}

Artigo recebido em 17/07/2018 e aceito em 17/08/2018

\begin{abstract}
RESUMO
Este trabalho propõe apresentar uma discussão teórico-prática de alguns elementos conceituais da geomorfologia fluvial, trazendo enfoque específico para o semiárido brasileiro. Na discussão, apresenta-se um panorama geral de como se dá o funcionamento desses ambientes, trazendo aplicações e exemplificações para os rios semiáridos, de modo específico para o rio Jaguaribe no Ceará, mostrando as particularidades e contradições. No geral observa-se que o funcionamento dos rios semiáridos apresenta fortes particularidades e que merecem ser melhor estudados, a fim de que se possa dirimir os efeitos de tantas alterações que vem sendo constatadas por uma série de obras que vem sendo implementadas no âbito de importantes bacias do Nordeste brasileiro.

Palavras-chaves: Geomorfologia Fluvial; Semiárido; Brasil.
\end{abstract}

\section{FLUVIAL GEOMORPHOLOGY IN THE BRAZILIAN SEMI-ARID}

\begin{abstract}
This work proposes to present a theoretical-practical discussion of some conceptual elements of fluvial geomorphology, bringing specific focus to the Brazilian semiarid. In the discussion, it is presented an overview of how the operation of these environments occurs, bringing applications and examples to the semi-arid rivers, specifically to the Jaguaribe river in Ceará State, showing the particularities and contradictions. In general, it is observed that the operation of the semi-arid rivers presents strong peculiarities and deserves to be better studied, in order to be able to resolve the effects of many changes that have been verified by a series of works that have been implemented in the context of important basins of the Brazilian Northeast.
\end{abstract}

Keywords: Fluvial Geomorphology; Semi-arid; Brazil.

\section{INTRODUÇÃO}

Grandes temas da geomorfologia fluvial vêm sendo trabalhados por uma série de autores importantes que estabeleceram uma base sólida de conceitos que vêm sendo trabalhados na atualidade. Tais trabalhos estão publicados em clássicos sob a forma de livros e artigos de veiculação importantes no mundo. Não podemos deixar de ressaltar que os conceitos relacionados aos fundamentos da geomorfologia fluvial tiveram forte contribuição dos trabalhos de Gilbert (1914), Mackin (1948), Leopold et al (1964), Gregory \& Walling (1973), Schumm (1977), Knighton (1998), Bridge (2003), Charlton (2008), além dos 
trabalhos de Christofolletti (1981), Cunha (1995) e Stevaux \& Latrubesse (2017), mais especificamente no Brasil.

Importantes contribuições nas discussões sobre padrões de canais, tema que ainda hoje traz grandes debates, foram dadas por Leopold \& Wolman (1957), Nanson \& Croke (1992), Knighton \& Nanson (1993), Nanson \& Knighton (19996), Xu (1996), Tooth \& Nanson (1999), Latrubesse (2008) entre outros. Porém, quando buscamos trabalhos relacionados à geomorfologia fluvial de áreas semiáridas, poucas contribuições são encontradas. Ainda assim, não podemos deixar de ressaltar que entre os trabalhos mais consistentes que vislumbram formas e processos específicos para os rios de áreas semiáridas, merecem destaque àqueles desenvolvidos por Graf (1988), Tooth (2000) e Bartley et al (2007). Fortes contribuições também foram dadas por Willians \& Wolman (1984), Brandt (2000) e Graf (2006) nas pesquisas sobre os impactos de barragens nos istemas fluvais, e mais recentemente, cabe destaque os trabalhos de Brierley et al (2005) nos estudos de gestão e estilos fluviais.

Entre tantos temas interessantes para a geomorfologia fluvial serão destacados neste trabalho alguns aspectos específicos da dinâmica fluvial de áreas semiáridas, trazendo elementos comparativos dessas áreas e outras áreas no mundo, a fim de mostrar as particularidades do funcionamento dos rios no semiárido brasileiro. Trazemos como exemplo um grande rio no Estado do Ceará (rio Jaguaribe), apresentando algumas diferenças em termos de variáveis hidrológicas, sedimentológicas e morfológicas (Figura 1).

Figura 1 - Localização da bacia do rio Jaguaribe-Ce, no Nordeste Brasileiro.

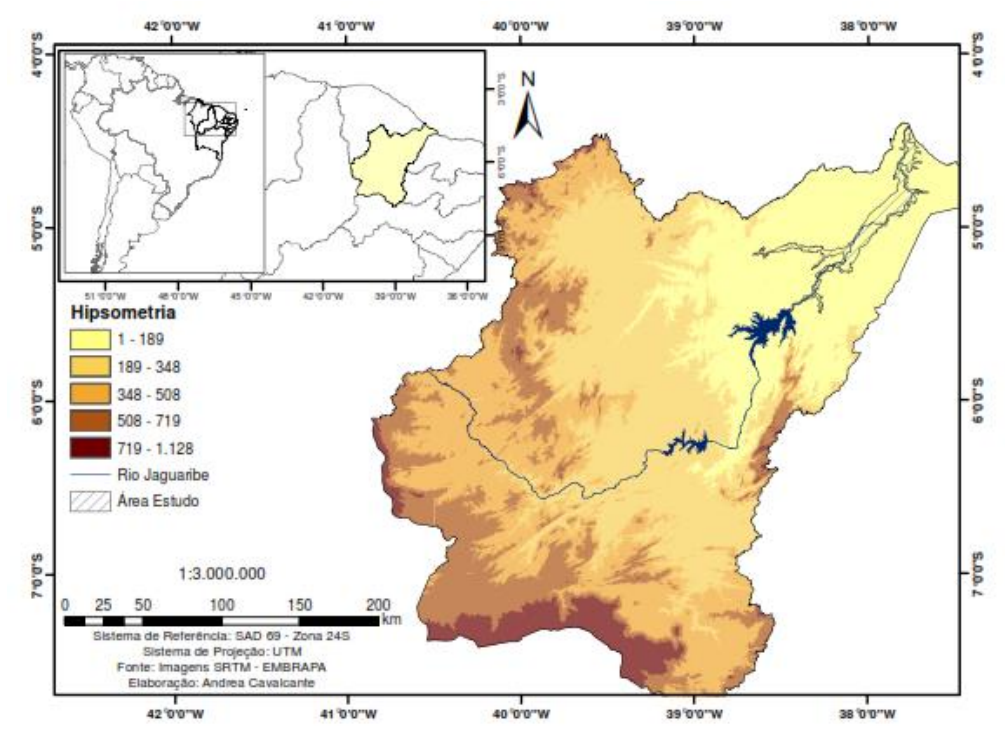




\section{MORFODINÂMICA DE CANAIS EM ÁREAS SEMIÁRIDAS: ELEMENTOS PARA UMA DISCUSSÃO}

A geometria de um sistema fluvial reflete um estado quase de equilíbrio entre vários fatores inter-relacionados. Alguns desses fatores como descarga, carga sedimentar e diâmetro dos sedimentos transportados, atuam de modo independente dentro do canal, pois são controlados por elementos externos, tais como, litologia e estrutura do substrato, relevo e clima (BIGARELLA e MOUSINHO, 1965; SCHUMM, 1977; BRIDGE, 2003). Em geral, descarga e carga de sedimentos controlam a forma de ajuste do canal (KNIGHTON, 1998). A declividade por sua vez, é um fator dependente, comumente ajustado aos processos atuantes dentro do canal. Outros fatores básicos dependentes incluem a largura e a profundidade, velocidade de fluxo e rugosidade do leito. As interações desses vários fatores constituem a geometria hidráulica do canal (SUGUIO e BIGARELLA, 1990).

Embora todas essas variáveis tenham um importante papel na dinâmica e comportamento de um canal fluvial, a descarga líquida ou vazão (Q) comumente representa uma variável de grande importância, sendo fundamental nos estudos fluvais. A vazão é, sem dúvida, umas das variáveis que mais dinamizam as áreas semiáridas, haja vista, estarem bem correlacionadas ao comportamento das precipitações que, nesse caso, caracterizam-se por serem mal distribuídas no tempo e no espaço, sendo sempre intensas e rápidas. Por essa razão as áreas semiáridas, geralmente experimentam, fluxos rápidos e intensos, sendo estes os maiores responsáveis pela intensa dinâmica vista nessas áreas.

No semiárido brasileiro as particularidades estão relacionadas ao modo como a intensidade e a frequência de precipitações atingem uma superfície de rala cobertura vegetal, como é a caatinga, a cada início de período chuvoso. Além disso, os diversos usos nas margens dos rios e a necessidade de acumulação de água mediante a construção de barragens de pequeno, médio e grande porte, também são fatores a serem considerados.

Tais diferenças podem ser claramente observadas na tabela 1 , onde dados de vazão e sedimentos para alguns rios no mundo são apresentados, denotando a importância do estudo dessas áreas, especialmente em razão do Nordeste Brasileiro ser também um dos mais povoados do mundo (Ab’Saber, 2003).

Em sentido espacial e temporal, os fluxos em rios semiáridos exibem ainda algumas características distintas. Porém, diferente de outras áreas semiáridas mundiais, cujos rios possuem fluxos sujeitos a uma diminuição à jusante (HUGHES e SAMI, 1992), podendo 
inclusive desaparecer (TOOTH, 2000b), o rio Jaguaribe (Nordeste Brasileiro) cresce, em fluxo, com a área de drenagem. Ressalta-se que no semiárido, esta diminuição de fluxo está relacionada, de modo essencial, as perdas por infiltração e evaporação.

Tabela 1 - Comportamento hidrológico e sedimentológico para alguns rios tropicais brasileiros e rios áridos e semiáridos mundiais.

\begin{tabular}{|c|c|c|c|c|c|c|c|c|}
\hline Rio & Estação & País & $\begin{array}{c}\text { Precipitação } \\
\left(\mathrm{mm} \mathbf{a n o}^{-1}\right)\end{array}$ & $\begin{array}{c}\text { Área } \\
\text { Drenagem } \\
\left(\mathbf{k m}^{2}\right)\end{array}$ & $\begin{array}{l}\text { Q Média } \\
\left(\mathrm{m}^{3} \mathrm{~s}^{-1}\right)\end{array}$ & $\begin{array}{l}\text { Css media } \\
\left(\mathrm{mg} \mathrm{L}^{-1}\right)\end{array}$ & $\begin{array}{c}\text { Qss } \\
\left(\mathbf{t} \mathbf{a n o}^{-1}\right)\end{array}$ & $\begin{array}{c}\text { Qss } \\
\left(\mathbf{t ~ k m}^{2} \text { ano }^{-1}\right)\end{array}$ \\
\hline Amazonas $^{1}$ & Óbidos & Brasil & 2.400 & 4.800 .000 & 172.000 & 149,33 & $810 \times 10^{6}$ & 168,8 \\
\hline Doce $^{2}$ & $\begin{array}{c}\text { Faz. } \\
\text { Cachoeira } \\
\text { D’Antas }\end{array}$ & Brasil & 1.500 & 10.080 & 161,1 & 197,58 & $1 \times 10^{6}$ & 99,6 \\
\hline Parnaíba $^{2}$ & Terezina & Brasil & 1.500 & 232.000 & 577,1 & 190,67 & $3,46 \times 10^{6}$ & 15 \\
\hline Iguaçu $^{2}$ & $\begin{array}{c}\text { Estreito } \\
\text { Iguaçu-Novo }\end{array}$ & Brasil & 1700-1900 & 63.236 & $1.767,9$ & 40 & $2,23 \times 10^{6}$ & 35,3 \\
\hline Tocantins $^{2}$ & Tucuruí & Brasil & 1800 & 742.300 & 10.981 & 8,84 & $3,06 \times 10^{6}$ & 4,1 \\
\hline Araguaia $^{3}$ & Aruanã & Brasil & $1500-2200$ & 77.000 & 1.200 & $20-200$ & $6,7 \times 10^{6}$ & 87 \\
\hline Isábena $^{4}$ & Capella & $\begin{array}{l}\text { Península } \\
\text { Ibérica** }\end{array}$ & 767 & 445 & 4,1 & $8-350$ & $1,84 \times 10^{5}$ & 414 \\
\hline Wadi Abdf ${ }^{5}$ & Ain Hamara & Algéria** & 250 & 2.480 & 1 & DND & $353 \times 10^{3}$ & 136 \\
\hline Rahaf $^{6}$ & Rahaf & Israel*** & $50-130$ & 78 & 18,5 & $1000-270.000$ & DND & DND \\
\hline Rio Piraí ${ }^{7}$ & Angostura & Bolivia** & $800-1300$ & 1.420 & 10 & 1.400 & $3 \times 10^{6}$ & 2.080 \\
\hline São Francisco ${ }^{2}$ & Própria & Brasil & 1200 & 623.500 & 2.120 & 27,38 & $1,83 \times 10^{6}$ & 2,9 \\
\hline Jaguaribe $^{8}$ & Iguatu & Brasil** & 700 & 20.673 & 79 & 114,99 & $2,1 \times 10^{6}$ & 100,6 \\
\hline Jaguaribe* & Icó & Brasil $^{* *}$ & 900 & 11.879 & 27,21 & 20,2 & $5 \times 10^{4}$ & 4,26 \\
\hline Jaguaribe* & Jaguaribe & Brasil $^{* *}$ & 700 & 39.800 & 60,64 & 17,41 & $4,3 \times 10^{4}$ & 1,09 \\
\hline Jaguaribe* & Peixe Gordo & Brasil** $^{*}$ & 700 & 48.200 & 97,9 & 19,9 & $7,4 \times 10^{4}$ & 1,55 \\
\hline
\end{tabular}

1- Guyot et al. (2005); 2- Lima et al. (2005); 3- Aquino et al. (2009); 4- López-Tarazón et al. (2009); 5- Achite e Ouillon (2007); 6- Cohen e Laronne (2005); 7- Guyot et al. (1994); 8- Wiegand (2009); *este estudo. Estação Peixe Gordo no período pré-barragem. ** área semiárida. ***área hiperárida. DND - dado não disponível. (Q) Vazão; (Css) Concentração de Sedimentos suspensos; (Qss) Descarga Sólida. 
Em relação ao transporte de sedimentos, faz necessário destacar que os estudos em rios de áreas semiáridas são restritos, sendo melhor documentados em rios de climas úmidos e temperados (REID e LARONNE, 1995). Em áreas semiáridas, a grande maioria destes concentram-se em pequenas bacias na Austrália Central (TOOTH, 2000b), Arizona-América do Norte (BEAUCHAMP et al., 2007), Espanha-Europa (LÓPEZ-TARAZON et al., 2009), Algéria-África (ACHITE et al., 2007), Israel-Ásia (COHEN e LARONNE, 2005) entre outras.

Tooth (2000b) afirma que áreas semiáridas podem ter altas taxas de concentração de sedimentos (30-50 $\left.\mathrm{g} \mathrm{L}^{-1}\right)$ e até mesmo exceder a $100 \mathrm{~g} \mathrm{~L}^{-1}$. Porém na bacia do rio Jaguaribe, os valores máximos encontrados não ultrapassaram $500 \mathrm{mg} \mathrm{L}^{-1}$, o que sugere que a carga de fundo possa ser predominante no total transportado (CAVALCANTE, 2012).

No cenário brasileiro é possível verificar claramente que o transporte de sedimentos em grandes rios é ditado pelas altas vazões, cuja média varia de $1.000 \mathrm{~m}^{3} \mathrm{~s}^{-1}$ (Araguaia) a $170.000 \mathrm{~m}^{3} \mathrm{~s}^{-1}$ (Amazonas), enquanto as taxas de concentrações, em geral, estão abaixo de $200 \mathrm{mg} \mathrm{L}^{-1}$ (Tabela 1).

De modo contrário às áreas tropicais, as áreas semiáridas observadas caracterizam-se por uma média de vazões inferiores, com concentrações bem superiores. Como visto na Tabela 1, áreas semiáridas e hiperáridas podem ter valores de concentração acima de $200 \mathrm{mg}$ $\mathrm{L}^{-1}$ (Península Ibérica) e $1.000 \mathrm{mg} \mathrm{L}^{-1}$ (Israel) em respectivo.

Outra variável importante que vem sendo estuadada é a energia do canal (stream power), haja vista as correlações existentes entre esta e o estabelecimento dos padrões de canais. Segundo Bridge (2003), a energia do canal (stream power), o tipo de grão e rugosidade do canal são variáveis dependentes e fundamentais na determinação de um padrão de canal.

Nesse sentido, a energia do canal $\Omega$ (stream power $)^{1}$ e a energia específica do canal $\omega$ (specific weight of the water) $^{2}$ tem sido estudadas como variáveis importantes no entendimento da morfologia de canais (SCHUMM, 1985; CHRISTOFOLETTI, 1981; GRAF, 1988; NANSON e CROKE, 1992; ROCHA, 2002; CHARLTON, 2008; LATRUBESSE, 2008) uma vez que envolve uma análise que correlaciona vazão de margens plenas,

\footnotetext{
${ }^{1}$ Expressão da taxa de energia potencial expedida por unidade de comprimento do canal.

${ }^{2}$ Energia do canal por unidade de largura.
} 
declividade e largura do canal, refletindo diretamente nas taxas de transporte e deposição, e por conseguinte, na forma do canal.

Nanson e Croke (1992) também atribuíram a energia específica do canal ( $\omega)$ e o tipo de sedimentos de fundo como variáveis importantes para o entendimento dos padrões de canais, sugerindo uma classificação interessante. De acordo com essa classificação o rio Jaguaribe seria considerado com um rio de média energia (stream power entre 10-300 W/m²),

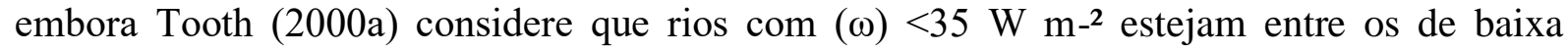
energia. Ao longo do leito deste rio predominam areias médias e grosseiras, podendo, ocasionalmente, ocorrer cascalho em áreas de maiores declividades. O padrão sugerido pelos autores para canais com tais características seria meandrante, porém este não se aplica na área, mostrando que os rios semiáridos podem apresentar muitas particularidades, carecendo de maiores estudos.

Em se tratando de padrões de canais, embora o padrão entrelaçado seja o mais comum encontrado em áreas semiáridas (GRAF, 1988; TOOTH, 2000a), estudos fluviais realizados na Austrália mostram que o padrão anabranching é passível de ser encontrado em áreas de rios efêmeros.

Tooth e Nanson (1999), estudando as implicações do transporte de sedimentos na morfologia de canais de rios efêmeros na Austrália Central, mostraram que tais rios podem alterar-se do padrão de um único canal (single-thread) para anabranching em resposta a entrada de água e sedimentos dos tributários durante eventos de enchentes, uma vez que tais rios possuem capacidade de transportar grande quantidade de sedimentos nesses fluxos. Para os autores, a formação de ilhas não estaria atrelada apenas à erosão mas, também, a grandes processos deposicionais gerados por fluxos excepcionais. Neste caso, faz necessário contextualizar que as áreas semiáridas, mesmo sendo caracterizadas, de modo predominante, pelas condições climáticas, precisam estar contextualizadas também em função de suas características litológicas e de declividade que são dois fatores importantes para maior ou menor produção de sedimentos.

Graf (1988) e Tooth (2000a) consideram que o padrão meândrico é mais difícil de ser encontrado em áreas semiáridas, sendo mais comuns em áreas úmidas, uma vez que são caracterizados por declives suaves, fluxos contínuos e regulares, e cargas em suspensão e fundo equilibradas (CUNHA, 1995). Entretanto, Graf (1988) observou em rios no sudoeste da América do Norte que os canais podem se apresentar de modo composto, ou seja, um canal 
meândrico pode estar inserido dentro de um grande canal entrelaçado. Neste caso, baixos fluxos ocupariam o canal meândrico menor, enquanto os altos fluxos seriam espalhados pelo canal maior designado como entrelaçado. Para o autor, os canais compostos podem ser uma forma de padrões estáveis em áreas semiáridas.

Entendendo que as áreas semiáridas experimentam grandes variações de descarga em curtos espaços de tempo, considera-se importante para essa pesquisa trabalhar com as ideias de padrões dos tipos compostos (GRAF, 1988; XU, 1996a), entrelaçados (GRAF, 1988; NANSON e CROKE, 1992; XU, 1996a; CHARLTON, 2008) e wandering gravel-bed (CARSON, 1984; KNIGHTON e NANSON, 1993; XU, 1996a; BRIERLEY e FRYIRS, 2005; e CHARLTON, 2008). Este último, ainda pouco discutido na atualidade, aparece como algo inovador na análise de áreas semiáridas, merecendo atenção para o desenvolvimento de maiores discussões nessas áreas.

Em termos gerais, observa-se que os maiores rios no semiárido Brasileiro são largos, rasos e de fundo arenoso. Além disso, as altas variações de descarga produzem a formação de uma diversidade de bancos arenosos ao longo de seus cursos.

Numa análise inicial, procurando aplicar essa discussão no semiárido cearense poderse-ia dizer que o rio Jaguaribe na maior parte do ano poderia ser caracterizado como um canal wandering, uma vez que é possível identificar um canal predominante em atividade que por vezes se divide, formando canais menores. Em águas mais elevadas, o rio poderia se espalhar pela calha fluvial formando outros canais, o que o aproximaria de um padrão mais próximo ao entrelaçado, porém sempre com a dominância de um dos canais.

Pensando o semiárido brasileiro, onde o índice de represamento é elevado, é possível que as vazões regularizadas mantidas pelos grandes barramentos, influenciem na mudança gradual dos padrões de canais até que estes alcancem novo equilíbrio. A transformação de um canal à jusante de um barramento geralmente é um processo longo e sua conclusão às vezes precisa de várias dezenas ou mesmo centenas de anos para ocorrer (XU, 1996b).

Baseado em relações estatísticas entre geometria de canal e fatores de descarga e sedimento, Schumm (1977) estabeleceu uma expressão frequentemente usada para prever a direção da mudança do canal após a construção de um reservatório. De acordo com este modelo, depois da construção do reservatório, a relação largura/profundidade (W/D) tende a diminuir, enquanto a sinuosidade do canal aumenta, e por isso o canal tende a meandrar. 
Porém, este modelo deve ser usado apenas para prever situação final da mudança, não sendo adequado seu uso em canais ainda em transição.

Segundo Vieira e Cunha (2008), a morfologia dos canais é bastante variável, já que a geometria é influenciada não somente pelo fluxo, mas pelo transporte e estabilidade das margens, incluindo estoque e liberação de água e sedimento dentro do sistema, modificando tamanho e forma do rio. Por essa razão, cada rio engendra um conjunto de características particulares, não podendo ser estudados por um modelo geral de alterações.

Do ponto de vista morfológico rios aluviais estabelecem padrões, que são, como citado, produto de interações de diversos fatores naturais. No conjunto, os padrões de canais refletem a hidrodinâmica de fluxo dentro do canal e os processos associados de transferência de sedimento e dissipação de energia (RICHARDS, 1982).

Assim, rios com diferentes padrões se comportam de modo diferente devido suas características morfo-hidráulicas. Por isso, a identificação destes padrões pode ser o primeiro passo para avaliar a estabilidade de um rio e identificar riscos potenciais (Schumm, 1985).

No cenário mundial, o semiárido brasileiro (representado pelo Jaguaribe) aparece com comportamento diferenciado dos demais. Embora também com uma média de vazões baixas, as concentrações quando comparadas às demais, praticamente desaparecem, o que sugere três caminhos para uma reflexão: alta descarga de fundo; alto índice de barramento nos rios da bacia; ou as características naturais da bacia fazem desta uma bacia de pouca produtividade de sedimentos.

Embora a maior parte dos estudos mostre que à jusante de barramentos o canal passe por ajustes, de modo especial pela erosão de fundo, vale ressaltar que a deficiência de sedimento provocada pelo barramento gerando tal erosão, pode ser suprida pelas margens (GALAY, 1983), e nesse caso, o ajuste se dará pelo alargamento destas ao invés da erosão pelo fundo.

Wolman e Gerson (1978) sugeriram que em sistemas fluviais de áreas áridas e semiáridas com vegetação esparsa, grandes enchentes podem produzir grandes efeitos geomórficos em razão de tais sistemas não possuírem vegetação suficiente para proteger as margens, fazendo destas áreas ambientes altamente sensitivos aos efeitos de grandes fluxos (TOOTH, 2000a). 
No rio São Francisco, Holanda et al. (2005) identificaram que margens com predominância de silte e argila, e predominância de grama e vegetação esparsa tiveram erosão em menor intensidade quando comparadas àquelas com predominância de silte e areia fina. Ainda assim, tais taxas $\left(4,45 \mathrm{~m}_{\text {ano-}}{ }^{1}\right)$ quando comparadas as da maioria dos rios em áreas temperadas $\left(0,06-2,4 \mathrm{~m} \mathrm{ano}^{-1}\right)$, conforme apresentado na Tabela 2, mostram que áreas semiáridas podem experimentar mudanças mais acentuadas no recuo ou aumento de margens, reposicionando o canal com maior facilidade.

Tabela 2 - Taxa de Recuo para rios em outras zonas climáticas.

\begin{tabular}{|c|c|c|c|c|}
\hline Rio/ localização & Clima & $\begin{array}{l}\text { Taxa de recuo } \\
\quad\left(\mathrm{m} \mathrm{ano}^{-1}\right)\end{array}$ & Método & Fonte \\
\hline Mississipi/ EUA & Subtropical & 23 & Mapas & Carey $(1969) *$ \\
\hline Brahmaputra/ Índia & Temperado & $6-275$ & Mapas & Coleman $(1969)^{*}$ \\
\hline Rio Ohio/ Kentucky/EUA & Temperado & 0,357 & Mapas & $\begin{array}{c}\text { Alexander e Nunally } \\
(1972)^{*}\end{array}$ \\
\hline Rio Beatton/ British Columbia & Temperado & 0,48 & $\begin{array}{l}\text { Árvores e } \\
\text { depósitos }\end{array}$ & $\begin{array}{l}\text { Hickin e Nanson } \\
\qquad(1975)^{*}\end{array}$ \\
\hline Missouri/ Dakota/EUA & Temperado & $1,7-7$ & Árvores & Everitti $(1968)^{*}$ \\
\hline Crawfordsburn/ Irlanda & Temperado & $0-0,5$ & Pinos & Hill (1973)* \\
\hline Clady/ Irlanda & Temperado & $0-0,064$ & Pinos & Hill (1973)* \\
\hline Torrens/ Austrália & Temperado & 0,58 & Pinos & Twidale (1964) \\
\hline Rio Exe/ Inglaterra & Temperado & $0,63-2,40$ & Pinos & Hooke (1980) \\
\hline Rio Creedy/ Inglaterra & Temperado & $0,26-0,52$ & Pinos & Hooke (1980) \\
\hline Rio Culm/ Inglaterra & Temperado & $0,18-0,63$ & Pinos & Hooke (1980) \\
\hline Rio Yarty/ Inglaterra & Temperado & $0,70-1,38$ & Pinos & Hooke (1980) \\
\hline Deer Creek/ Ontario/ Canadá & Temperado & $0,21-0,61$ & Perfis & Buma e Day (1977) \\
\hline São Francisco/ NE/ Brasil & Semiárido & $4,45-47,3$ & Pinos & Casado et al. (2002) \\
\hline Rio Jaguaribe/ NE/ Brasil & Semiárido & $0,05-23$ & Pinos & Este estudo \\
\hline
\end{tabular}

*Retirado de Hooke (1980). 
Apesar dos processos relacionados à participação da vegetação nas alterações da morfologia de canais sejam pouco conhecidos (THORNES, 1994), muitos canais em áreas semiáridas têm mostrado serem suscetíveis aos efeitos erosivos de grandes inundações, devido à relativa escassez de vegetação (WOLMAN e GERSON, 1978). Mesmo os canais bem vegetados (com árvores, arbustos e gramas) são, algumas vezes, sujeitos à mudanças relacionadas à inundações em razão da concentração da vegetação próxima ao canal principal (TOOTH, 2000a). Em contraste, em áreas temperadas e tropicais úmidas a vegetação não pode agir como principal fator morfogenético na escala do corredor fluvial (CORENBLIT et al., 2007).

Como pode ser visto, as áreas semiáridas parecem experimentar fortes mudanças em curtos espaços de tempo, pelas condições naturais que apresentam. Esse fato nos faz pensar que o entendimento do funcionamento desses ambientes pode ajudar a dirimir tantos problemas que cotidianamente são visualizados nessas áreas, principalmente por estarem sendo alvo, nas últimas décadas, de grandes empreendimentos.

O que observamos é que embora o semiárido brasileiro ocupe cerca de 1/5 do território nacional, sendo o Nordeste um dos semiáridos mais ocupados do planeta, verifica-se que ainda hoje a carência de estudos aplicados para essas áreas ainda se faz presente, o que traz grandes preocupações, haja vista que são nessas mesmas áreas que os maiores projetos de desenvolvimento estão acontecendo e os efeitos de tantas intervesões não estão sendo vislumbrados a contento. Este é o caso da instalação de grandes barragens e da interligação de bacias como é o caso da transposição do rio São Francisco, que além de criar um sistema canais artificiais para ligar essas águas, integrará também o chamado Cinturão das Águas no Ceará. Tais intervenções têm modificado fortemente esses ambientes, levando os sistemas fluviais a procurarem novas formas de equilíbrio, as quais muitas vezes não são alcançadas.

Entre os problemas evidenciados no âmbito da transposição das águas do São Francisco, tanto nas bacias doadoras como receptoras, cabe destacar uma série de impactos socioambientais que já vem sendo discutidos como é o caso da erosão das margens dos rios e consequente assoreamento do canal fluvial, as ocupações irregulares, poluição, industrialização, agronegócio, privatização do recurso hídrico, carcinicultura, dentre outros imponentes impactos causados pelo avanço do desenvolvimento capitalista. 


\section{CONSIDERAÇÕES FINAIS}

A dinâmica fluvial em áreas áridas e semiáridas diferencia-se de outras climáticas como as tropicais e temperadas, refletindo padrões de comportamento particulares. $\mathrm{Na}$ América do Sul, em especial no Brasil, estudos sobre o comportamento de canais no semiárido ainda são pouco discutidos, mesmo diante do grande número de intervenções observado nas bacias de drenagem nordestinas.

Esse trabalho procurou trazer uma discussão teórico-prática de alguns temas discutidos na geomorfologia fluvial, apresentando a fragilidade de aplicação de alguns conceitos para os rios semiáridos, e ao mesmo tempo procurando mostrar que as particularidades desses rios necessitam ser melhor estudadas, para que o entendimento do seu funcionamento possa ajudar a pensar melhor que tipos de efeitos podem ser esperados diante da implementação de grandes obras como as que vem sendo implementadas no Nordesde do Brasil, de modo especial as grandes barragens e a transposição do São Francisco.

\section{REFERENCIAS}

AB'SÁBER, A.N. 2003. Os Domínios de Natureza no Brasil-potencialidades paisagísticas. São Paulo : Ateliê Editorial, 2003. 159p.

ACHITE, Mohamed; OUILLON, Sylvain. Suspended sediment transport in a semiarid watershed, Wadi Abd, Algeria (1973-1995). Journal of Hydrology, v. 343, p.187-202, 2007. AQUINO, S.; LATRUBESSE, E.; BAYER, M. Assessment of wash load transport in the Araguaia river (Aruanã gauge station), central brazil. Latin American Journal of Sedimentology and Basin Analysis, 16 (2), p.119-128, 2009.

BARTLEY, R.; HAWDON, A.; POST, D.A.; ROTH, C.H. A Sediment Budget for a Grazed Semi-Arid Catchment in the Burdekin basin, Australia. Geomorphology. № 87, p.302-321, 2007.

BEAUCHAMP, V B; STROMBERG, J.C.; STUTZ, C. Flow regulation has minimal influence on mycorrhizal fungi of a semi-arid floodplain ecosystem despite changes in hydrology, soils, and vegetation. Journal of Arid Environments 68, p.188-205, 2007.

BIGARELlA, J.J.; MOUSINHO, M.R. Considerações a respeito dos terraços fluviais, rampas de colúvios e várzeas. Boletim Paranaense de Geociências. №16/17, Curitiba, p.153197, 1965. 
BRANDT, S. Anders. Classification of Geomorphological effects downstream of Dams. Catena, $\mathrm{n}^{\circ} 40$, p. $375-401,2000$.

BRIDGE, J. S. Rivers and floodplains: forms, processes, and sedimentary record. 2003. 491p. BRIERLEY, G.J.; FRYIRS, K. A. Geomorphology and River Management : applications of the river styles framework. Oxford: Blackwell Oxford. 2005. 398p.

BUMA, P.G.; DAY, J.C. Channel Morphology Below Reservoir Storage Projects. Environmental Conservation. 4 (04), p.279-284, 1977.

CARSON, M.A. Observations on the Meandering-Braided River Transition, The Canterbury Plains, New Zealand: Part Two. New Zealand Geographer, 40 (2), p.89-99, 1984.

CASADO, A. P. B., HOLANDA, F. S. R., e YAGUIU, P. Evolução do processo erosivo na margem direita do rio São Francisco (perímetro irrigado Cotinguiba /Pindoba - SE. Revista Brasileira de Ciência do Solo, 26(1), p.231-239, 2002.

CAVAlCANTE, A.A. Morfodinâmica fluvial em áreas semiáridas: o Rio Jaguaribe a jusante da Barragem do Castanhão-Ceará-Brasil. Niterói: Universidade Federal Fluminense, 2012. 249p. Tese de doutorado, Programa de Pós-Graduação em geografia. Niterói, 2012.

CHARLTON, R. Fundamentals of Fluvial Geomorphology. Routledge. London and New York. 2008. 234p.

CHRITOFOLETTI, A. Geomorfologia Fluvial. São Paulo: Edgard Blücher, 1981. 312p.

COHEN, H.; LARONNE, J.B. High rates of sediment transport by flashfloods in the Southern Judean Desert, Israel. Hydrological Processes. 19 (8), p.1687-1702, 2005.

CORENBLIT, D., TABACCHI, E., STEIGER, J., e GURNELL, A. M. Reciprocal interactions and adjustments between fluvial landforms and vegetation dynamics in river corridors: A review of complementary approaches. Earth-Science Reviews. 84(1-2), p.56-86, 2007.

CUNHA, S.B. da. Geomorfologia Fluvial. In: GUERRA, A.J.T.; CUNHA, S.B. (orgs.) Geomorfologia uma Atualização de Bases e Conceitos. $4^{\mathrm{a}}$ ed. Rio de Janeiro: Bertrand Brasil, 1995. p. 211-252.

GALAY, V. J. Causes of river bed degradation. Water Resources Research, (19), p.10571090, 1983.

GILBERT, G. K. The transportation of debris by runnung water. Washington: U.S. Geol. Survey Professional Paper 86. 1914. 263p. 
GRAF, W. Fluvial Processes in Dryland Rivers. The Blackburn Press. New Jersey, USA, 1988. 346p.

GREGORY, K.J.; WALLING, D.E. Drainage Basin: form an process - a geomorpholocal approach. London, Edward Arnold, 1973. 458p.

GUYOT, J.L.; BOURGES, J. Sediment transport in the Rio Grande, an Andean river of the Bolivian Amazon drainage basin. In: Variability in Stream Erosion and Sediment Transport, ed. Proceedings of the Camberra Symposium, IAHS Publication, p. 223-231, 1994.

GUYOT, J.L.; FILIZOLA, N.P.; LARAQUE, A. Régime et bilan du flux sédimentaire de l'Amazone à Óbidos (Pará, Brésil) de 1995 à 2003. In: Sediment budgets 1. WALLING, D.E.; HOROWITZ, A.J. (Ed.) Oxfordshire: IAHS. p.347-354, 2005.

HOOKE, J. Magnitude and distribution of rates of river bank erosion. Earth Surface Processes, 5, p.143-157, 1980.

HUGHES, D.A., SAMI, K. Transmission losses to alluvium and associated moisture dynamics in a semi-arid ephemeral channel system in southern Africa. Hydrological Processes 6, p.45-53, 1992.

KNIGHTON, A.D.; NANSON, G.C. Anastomosis and the Continuum of Channel Pattern. Earth Surface Processes and Landforms. 18, 1993. p.613-625.

KNIGHTON, A.D.; NANSON, G.C. Waterholes and their significance in the anastomosing channel system of Cooper Creek, Australia. Geomorphology, 9 (4), p.311-324, 1994.

KNIGHTON, D. Fluvial Forms and Processes: a new perspective. London: Arnold. 1998. $383 p$.

LATRUBESSE, E.M. Patterns of anabranching channels: The ultimate end-member adjustment of mega rivers. Geomorphology, v.101, p.130-145, 2008.

LEOPOLD, L.B.; WOLMAN, M.G. River Channel Patterns: Braided, Meandering and Straight. U.S. Geological Survey Professional Paper. 282-B,1957. 51p.

LEOPOLD, L.B.; WOLMAN, M.Gordon; MILLER, John P. Fluvial Processes in Geomorphology. S. Chand and Company LTD. Ram Nagar, New Delhi-55, 1964. 536p.

LIMA, J.E.F.W.; LOPES, W.T.A.L.; CARVALHO, N.O.; SILVA, E.M; VIEIRA, M.R. Suspended sediment fluxes in the large river basins of Brazil. In: Sediment Budgets 1, ed. D.E. WALLING and A.J. HOROWITS. IAHS Publication. p.355-363, 2005.

LÓPEZ-BERMUDEZ, F.; CONESA-GARCIA, C.; ALONSO-SARRIA, F. Floods: Magnitude and Frequency in Ephemeral Streams of the Spanish Mediterranean Region. In: 
LÓPEZ-TARAZON, J.A.; BATALLA, R.J.; VERICAT, D.; FRANCKE, T. Suspended sediment transport in a highly erodible catchment: The River Isábena (Southern Pyrenees). Geomorphology. 109 (3-4), p. 210-221, 2009.

MACKIN, J. Hoover. Concept of the Graded River. Bulletin of the Geological Society of America. Vol. 59, p. 463-512,1948.

NANSON, G.C.; CROKE, J.C. A genetic classification of floodplains. Geomorphology. 4, p.459-486, 1992.

NANSON, G.C.; KNIGHTON, A.D. Anabranching Rivers: their cause, character ad classification. Earth Surface Process and Landforms. 21, p.217-239, 1996.

REID, I.; LARONNE, J.B. Bed load sediment transport in an ephemeral stream and a comparison with seasonal and perennial counterparts. Water Resources Research. 31 (3), p.773-781, 1995.

REID, I.; LARONNE, J.B.; POWELL, D.M. Flash-flood and bedload dynamics of desert gravel-bed streams. Hydrological Processes. 12 (4), p. 543-557, 1998.

RICHARDS, K. Rivers - Form and Processes in alluvial channels. New Jersey: The Blackburn Press, 1982. 361p.

SCHUMM, S.A. Explanation and Extrapolation in Geomorphology: seven reasons for geologyc uncertainty. Tansactions Japonese Geomorphological Union. № 6(1), 1985. p.1-18. SCHUMM, S.A. The Fluvial System. Ed. The Blackburn Press. Colorado: Press, The Blackburn. 1977. 338p.

STEVAUX, J.C.; LATRUBESSE, E.M. Geomorfologia Fluavial. São Paulo: Oficina de Textos, 2017.

THORNES, J. B. Channel processes, evolution and history. In A. D. ABRAHAMS e A. J. PARSONS (Eds.), Geomorphology of Desert Environments. London: Chapman and Hall, 1994. p. 288-317.

TOOTH, S. Downstream changes in dryland river channels: the Northern Plains of arid central Australia. Geomorphology, 34, p.33-54, 2000 b.

TOOTH, S. Process, form and change in dryland rivers: a review of recent research. EarthScience Reviews, 51, p.67-107, 2000a.

TOOTH, S.; NANSON, G. C. Anabranching rivers on the Northern Plains of arid central Australia. Geomorphology, 29, p.211-233, 1999. 
VIEIRA, V.T.; CUNHA, S.B da. Mudanças na Morfologia dos Canais Urbanos: alto curso do rio Paquequer, Teresópolis _ RJ (1997/98 - 2001). Revista Brasileira de Geomorfologia. V. 9, $\mathrm{n}^{\mathrm{o}} 1$, p. 3-23, 2008.

WILLIAMS, G. P.; WOLMAN, M. G. Downstream effects of dams on alluvial rivers. United States Geological Survey Professional Paper, (1286), 83, 1984.

WOLMAN, M. G. e GERSON, R. Relative scales of time and effectiveness of climate in watershed geomorphology. Earth Surface Processes and Landforms, 3, p.189-208, 1978.

XU, J. Channel pattern change downstream from a reservoir: An example of wandering braided rivers. Geomorphology, 15, p.147-158, 1996 b.

$\mathrm{XU}$, J. Wandering braided river channel pattern developed under quasi-equilibrium: an example from the Hanjiang River, China. Journal of hydrology, 181 (1-4), p. 85-103, 1996a. 